\title{
A Colorimetric CMOS-Based Platform for Rapid Total Serum Cholesterol Quantification
}

\author{
Mohammed A. Al-Rawhani, Boon Chong Cheah, Alasdair Iain Macdonald, Christopher Martin, Chunxiao Hu, \\ James Beeley, Luiz Carlos Gouveia, James P. Grant, Gordon Campbell, Michael P. Barrett, \\ and David R. S. Cumming, Fellow, IEEE
}

\begin{abstract}
Elevated cholesterol levels are associated with a greater risk of developing cardiovascular disease and other illnesses, making it a prime candidate for detection on a disposable biosensor for rapid point of care diagnostics. One of the methods to quantify cholesterol levels in human blood serum uses an optically mediated enzyme assay and a bench top spectrophotometer. The bulkiness and power hungry nature of the equipment limits its usage to laboratories. Here, we present a new disposable sensing platform that is based on a complementary metal oxide semiconductor process for total cholesterol quantification in pure blood serum. The platform that we implemented comprises readily mass-manufacturable components that exploit the colorimetric changes of cholesterol oxidase and cholesterol esterase reactions. We have shown that our quantification results are comparable to that obtained by a bench top spectrophotometer. Using the implemented device, we have measured cholesterol concentration in human blood serum as low as $29 \mu \mathrm{M}$ with a limit of detection at $13 \mu \mathrm{M}$, which is approximately 400 times lower than average physiological range, implying that our device also has the potential to be used for applications that require greater sensitivity.
\end{abstract}

Index Terms-Biosensor, CMOS, colorimetric, diagnostics, enzyme, photodiode.

\section{INTRODUCTION}

$\mathbf{P}$ OINT of care diagnostics are transforming the healthcare industry, by facilitating the use of home-testing to provide an early indication of potential illness and disease [1]-[3]. The development of low-cost and effective consumable biosensors [4], [5] is at the forefront of the research for userorientated testing, driven in part by the need for rapid diagnosis and monitoring without overburdening the resources of the healthcare services. For example, glucose biosensors, have

Manuscript received August 4, 2016; revised November 9, 2016; accepted November 9, 2016. Date of publication November 15, 2016; date of current version December 20, 2016. This work was supported by the U.K. Engineering and Physical Sciences Research Council under Grant EP/K021966/1. All our dataset can be accessed here: http://dx.doi.org/10.5525/gla.researchdata.371. The associate editor coordinating the review of this paper and approving it for publication was Prof. Sang-Seok Lee.

M. A. Al-Rawhani, B. C. Cheah, C. Martin, C. Hu, J. Beeley, L. C. Gouveia, J. P. Grant, and D. R. S. Cumming are with the Microsystems Technology Group, School of Engineering, University of Glasgow, Glasgow, G12 8LT, U.K. (e-mail: mohammed.al-rawhani@glasgow.ac.uk).

A. I. MacDonald and G. Campbell are with the Wellcome Trust Centre for Molecular Parasitology, Institute of Infection, Immunity and Inflammation, University of Glasgow, Glasgow, G12 8TA, U.K.

M. P. Barrett is with the Wellcome Trust Centre for Molecular Parasitology, Institute of Infection, Immunity and Inflammation, University of Glasgow, Glasgow, G12 8TA, U.K., and also with Glasgow Polyomics, University of Glasgow, East Dunbartonshire, G61 1BD, U.K.

Digital Object Identifier 10.1109/JSEN.2016.2629018 become widespread in their use for managing diabetes [6]. Furthermore, metabolite biomarkers of different diseases are also becoming increasingly well understood [7], [8] which paves the way for developing new diagnostic system. Here we report on the development of an electronic device that is capable of detecting cholesterol whose variable concentrations are predictive of cardiovascular diseases development [9]. We anticipate that future development will contribute to the introduction of stratified and precision medicine.

Elevated cholesterol levels are well known for their association with an increased risk of coronary heart disease (angina or heart attack), narrowing of the arteries (atherosclerosis), stroke, peripheral heart disease, and hypertension [10], [11]. Such conditions often correlated with poor diet, an excessive fat intake, lack of exercise and other lifestyle choices [12]. This pose a global threat to public health and consume considerable healthcare resources [13]. Hence, cholesterol diagnosis has already entered systematic use in many healthcare systems with prescription of cholesterol lowering drugs e.g. statins. It is estimated that approximately 73.5 million adults have high amounts of LDL* cholesterol in the US alone [14] and approximately 17 million deaths a year, nearly one third of the total [15], arise through cardiovascular disease.

One of the diagnostic methods for quantifying cholesterol concentration depends on enzyme-based assays that require a classical spectrophotometer to measure changes in colour products from those enzyme reactions [16]. A general purpose spectrophotometer would incorporate a sophisticated setup of a white light source, a monochromator containing a diffraction grating and a light transducer that converts light into electrical signal such as a charge-coupled device (CCD), a photodiode or a photomultiplier tube [17]. The wide spectrum range of the spectrophotometer makes it bulky and power hungry which consequently confines its usefulness to laboratories and hospitals.

The working principle of a spectrophotometer to measure colour changes of enzyme assay depends on setting up the monochromator's output light to a specific wavelength that matches the assay's absorption peak wavelength. By using the same principle, in this work we propose a disposable sensing platform that is designed to operate using a single wavelength. By careful selection of the right combination of enzyme absorption wavelength, light source wavelength and detector spectrum, a simpler-disposable version of the spectrophotometer can be implemented using components that can be industrially mass-produced. 
The platform that we have implemented comprises a complementary metal oxide semiconductor (CMOS)-based photodiode array and an off-the-shelf light emitting diode (LED). The photodiode array is fabricated using commercial standard CMOS process, which is readily available for low-cost mass-production [18].

Photodiodes made in a CMOS process are generally sensitive to light in the $200 \mathrm{~nm}$ to $1100 \mathrm{~nm}$ range, owing to the bandgap of silicon $(1.12 \mathrm{eV})$. This range makes them suitable for colorimetric enzyme assays that use visible light or fluorescent mediators, which often use wavelengths in the range $400 \mathrm{~nm}$ to $700 \mathrm{~nm}$. A colour change in this range of enzyme assays, e.g. cholesterol ester hydrolase [19], cholesterol dehydrogenase [20], cholesterol esterase [21] and cholesterol oxidase [22] can be exploited to extract cholesterol levels. In this work, we have employed cholesterol oxidase and cholesterol esterase assay to quantify total cholesterol from human blood serum samples.

\section{Materials AND Methods}

\section{A. Assay Materials}

Cholesterol oxidase from Streptomyces (C8649, 23 Units/mg solid) was purchased from Sigma-Aldrich, as was cholesterol (C3045), o-Dianisidine dihydrochloride (F5803), triethanolamine hydrochloride (T9534), Triton ${ }^{\mathrm{TM} X}-100$ (T9284) and horseradish peroxidase (P8250, 163 Units/mg solid). Cholesterol esterase from Pseudomonas fluorescens (C9281-5 Units/mg solid) was purchased from SigmaAldrich as was taurocholic acid sodium salt hydrate (T4009) and sodium cholate hydrate (C1254). Sodium chloride was purchased from VWR (27810). Human serum from human male AB plasma, USA origin, sterile-filtered was sourced from Sigma Aldrich (H4522). The serum cholesterol concentration was stated as $110-210 \mathrm{mg} / \mathrm{dl}$.

\section{B. Enzyme Assay Methods}

Approximately $30 \%$ of cholesterol in serum is present in 'free' form (typically, $40 \mathrm{mg} / \mathrm{dl}-85 \mathrm{mg} / \mathrm{dl}$ ) while the remainder is assimilated into cholesteryl esters with long-chain fatty acids [23]. In order to measure the total cholesterol in serum, a triple enzyme assay was used comprising two cholesterol reactions and an optical product step via the activity of peroxidase. First, the enzyme cholesterol esterase was used to hydrolyse cholesterol esters to free cholesterol [24]. The reaction is as below:

$$
\begin{aligned}
\text { CholesterylEster }+\mathrm{H}_{2} \mathrm{O} \stackrel{\text { CholesterolEsterase }}{\longrightarrow} & \text { Cholesterol } \\
& + \text { FattyAcid }
\end{aligned}
$$

The second reaction involved the addition of cholesterol oxidase, which is an oxidoreductase that uses molecular oxygen as an electron acceptor to reduce oxygen to hydrogen peroxide [25]. In this reaction, free cholesterol in serum with the addition of free cholesterol from cholesterol esterase reaction is used by the cholesterol oxidase, as a mean of quantification. The cholesterol oxidase catalysed reaction is

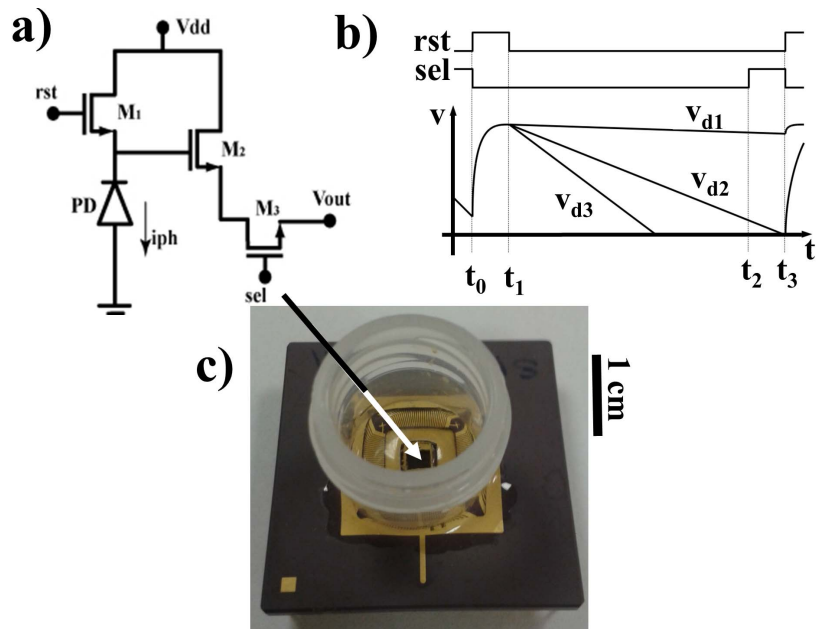

Fig. 1. a) Circuit diagram of a single photodiode pixel. b) Illustrative waveforms showing how the output voltage responds to three different light levels. The light intensity is greatest for the curve labeled $\mathrm{V}_{\mathrm{d} 3}$. c) Packaging of the CMOS chip for aqueous measurements. The chip is $3.4 \mathrm{~mm} \times 3.6 \mathrm{~mm}$ with an active area of $1.6 \mathrm{~mm} \times 1.6 \mathrm{~mm}$.

as below:

$$
\begin{aligned}
\text { Cholesterol }+\mathrm{O}_{2} \stackrel{\text { CholesterolOxidase }}{\longrightarrow} & 4-\text { Cholesten }-3 \\
& - \text { one }+\mathrm{H}_{2} \mathrm{O}_{2}
\end{aligned}
$$

The third reaction involved the production of a coloured product to be measured optically. In the presence of $\mathrm{H}_{2} \mathrm{O}_{2}$ produced from cholesterol oxidase, o-Dianisidine is oxidised to a coloured product with a progressively deep orange colour, amenable to detection by absorbance of green light. The intensity of the coloured product is dependent on the concentration of $\mathrm{H}_{2} \mathrm{O}_{2}$, which is proportional to cholesterol concentration.

$$
\begin{aligned}
& \stackrel{o-\text { Dianisidine }(\text { reduced })+\mathrm{H}_{2} \mathrm{O}_{2}}{\stackrel{\text { Peroxidase }}{\longrightarrow} o-\text { Dianisidine }(\text { oxidised })+\mathrm{H}_{2} \mathrm{O}}
\end{aligned}
$$

Therefore, these three enzyme reactions allow for a colorimetric measurement of total cholesterol in serum.

\section{CMOS Chip}

An integrated circuits chip was designed using Cadence Design Software version 5.10, and fabricated in the AMS $0.35 \mu \mathrm{m}$ CMOS process provided by Austriamicrosystems. The chip incorporates an array of $16 \times 16$ photodiodes with integrated addressing and readout circuits. Each chip measured $3.4 \mathrm{~mm}$ by $3.6 \mathrm{~mm}$, with an active sensor array area of $1.6 \mathrm{~mm}$ by $1.6 \mathrm{~mm}$. The photodiode pixel used for these experiments measured $10 \mu \mathrm{m} \times 24 \mu \mathrm{m}$.

In this chip we used a typical active pixel sensor (APS) design that incorporates a photodiode and three transistors to enable the readout of data [26]. The schematic of a single pixel is shown in Fig. 1a. As can be seen in the timing diagram of Fig. 1b, each measurement cycle begins at $t=t_{0}$ when $V_{r s t}=V_{d d}$. From $t_{0}$ to $t_{1}$ the junction capacitance of the diode is precharged to be $V_{d d}$. At $t=t_{1}$ the circuit enters the integration phase. Depending on the integration time, a different photocurrent $\mathrm{I}_{\mathrm{ph}}$ corresponds to a change of the voltage, $\mathrm{V}_{\mathrm{d}}$, across the diode. At $\mathrm{t}=\mathrm{t}_{2}$ the diode voltage is 
a)

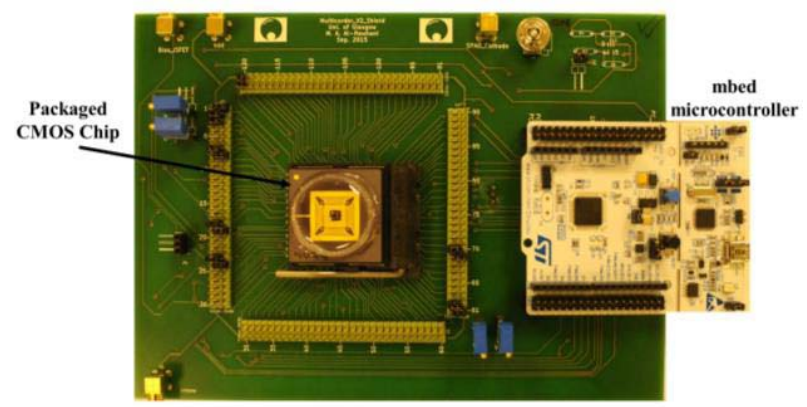

b)

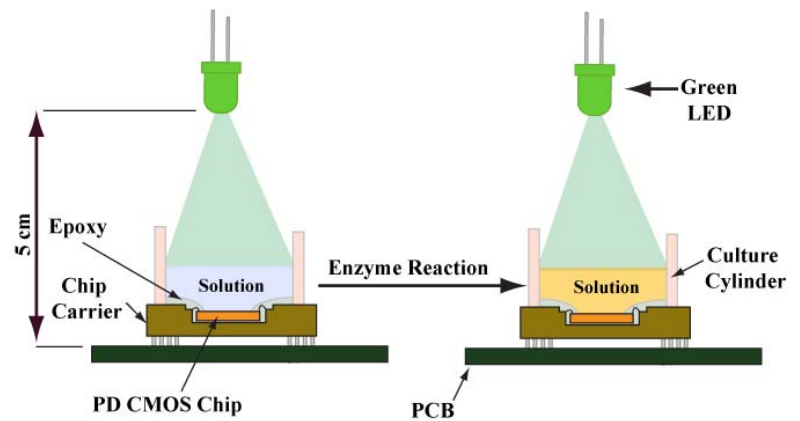

Fig. 2. a) The implemented diagnostic platform incorporates a CMOS photodiode array chip and mbed microcontroller. b) Schematic of the experimental set up. A green LED provides the excitation light, which is collected by the photodiodes. As cholesterol oxidase catalyzes the cholesterol reaction, the oxidation of o-Dianisidine results in fewer photons from the LED reaching the photodiode and hence a change in voltage from the chip directly infers the enzyme activity and thus the amount of cholesterol present in solution.

sampled and read-out using the 'sel' signal on M3. As can be seen from Fig. $1 b$ the sampled voltage decreases as the light intensity is increased (e.g. for traces labelled $V_{\mathrm{d} 1}$ and $\mathrm{V}_{\mathrm{d} 2}$ ). However, if the detector is saturated, the diode will have completely discharged before $t=t_{2}$. This is illustrated by the trace labelled $\mathrm{V}_{\mathrm{d} 3}$.

Fig. 1c shows the CMOS chip after packaging. The chip was packaged by attaching it to a chip carrier using EPO-TEK H74 epoxy (Epoxy Technology Inc.). The epoxy, heated to $150^{\circ} \mathrm{C}$, formed a strong bond between the chip and the carrier. A wirebonding machine was then used to make the connections between the chip and chip carrier. Next, a $2 \mathrm{~mm}$ cube of polydimethylsiloxane (PDMS) was placed on the sensor active area as protection before adding EPO-TEK 302-3M (Epoxy Technology Inc.) to protect the bond-wires. This epoxy was cured at room temperature for duration of 24 hours. The PDMS was then removed from the sensor surface before cleaning the sensor with acetone and isopropanol. Finally, a section of polypropylene centrifuge tube (Fisherbrand) was attached with more EPO-TEK $302-3 \mathrm{M}$ and cured at $65^{\circ} \mathrm{C}$ for 3 hours to allow for aqueous measurements to occur on the chip surface.

\section{Experimental Setup}

In order to operate the photodiode array chip, a printed circuit board (PCB) platform was designed to integrate the chip with an ARM mbed STM32 Nucleo-F334R8 board (STMicroelectronics, UK) as shown in Fig. 2a. The mbed microcontroller was programmed to provide addressing signals

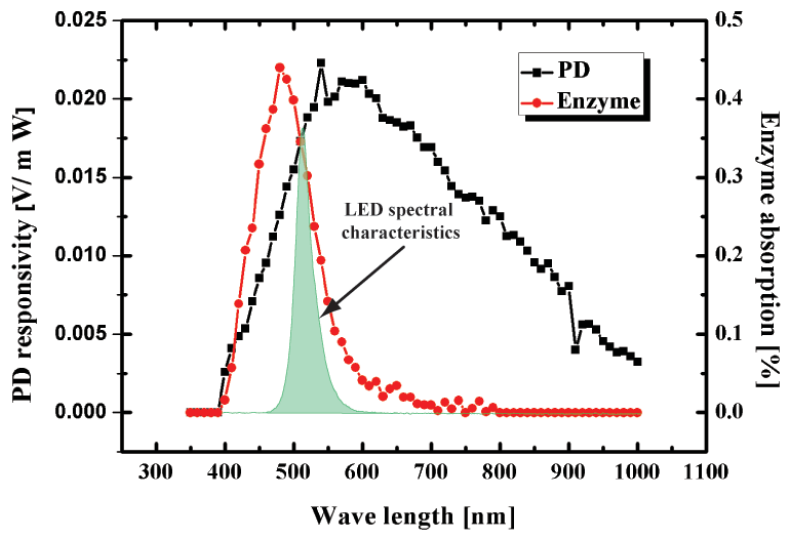

Fig. 3. The spectral response of the photodiode, the enzyme solution after a reaction has occurred, and the green LED emission characteristics. The peak in the LED emission intensity occurs very near the optimum in the overlap in the enzyme absorbance and PD sensitivity spectra.

and to acquire the output readings from the array. These readings were then transferred by a universal serial bus (USB) to a computer based LabVIEW program, in which the data was processed and analysed. Fig. $2 \mathrm{~b}$ shows the concept of utilising the experimental setup in conjunction with a LED to quantify the coloured product from the enzyme assay.

\section{EXPERIMENTAL RESULTS AND DISCUSSIONS}

\section{A. Optical Evaluation of the System}

The optical characteristics of the photodiode and a completed cholesterol reaction mix, were evaluated to examine their spectral relationship. First, the spectrum of the photodiode was measured using a TMc300 monochromated light source (Benthem Instruments), swept between $350 \mathrm{~nm}$ and $1000 \mathrm{~nm}$. The photodiode responsivity shown in Fig. 3 has a maximum peak at c. $600 \mathrm{~nm}$. The enzyme spectrum was then measured using a TFProbe MSP300 spectrometer (Angstrom Sun Technologies) after all the substrate had been consumed. The measurement showed that the enzyme spectrum has an absorption peak at c. $460 \mathrm{~nm}$. From both traces, it can be seen that there is an overlap between sensitivity of the photodiode and the absorbance spectrum of the enzyme assay, around $500 \mathrm{~nm}$. Based on this data, a green LED was chosen to have a peak spectrum close to $500 \mathrm{~nm}$. An off-the-shelf commercial green LED was measured using the same spectrometer and is shown to have a peak at $505 \mathrm{~nm}$, which was well positioned at the overlap of the two traces.

\section{B. Electrical Evaluation of the System}

In this section, we present the electrical characteristics of the photodiode array, in terms of dynamic range and noise performance. The photodiode array has a minimum light detection of $280 \mathrm{nW} / \mathrm{cm}^{2}$ and a total dynamic range of $56 \mathrm{~dB}$. In terms of noise, the chip was designed to have an array of 256 sensors, which had proven to be able to outperform a single sensor. In our previous work, we had demonstrated that an array of ion sensitive field effect transistor (ISFET) sensors could be used as an average to reduce noise [27]. 


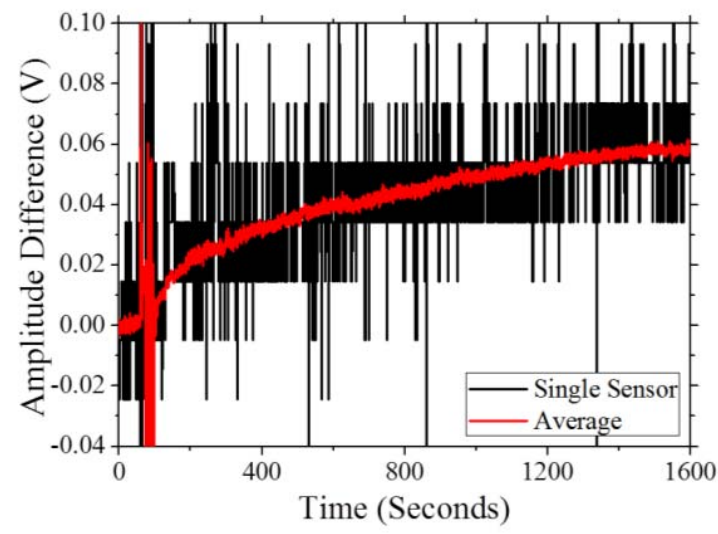

Fig. 4. Comparison of the readout signal from a typical pixel and the average readout of the $16 \times 16$ array.

In the same way, the photodiode array was used to reduce the noise of the combined assay signal, since we can obtain data from a single photodiode, or we can average data from 2 to $2^{8}$ photodiodes. Following Gaussian statistics, the noise is reduced as a function of $\sqrt{N}$, where $\mathrm{N}$ is the number of sensors. Fig. 4 illustrates a typical time-course signal obtained from the chip for a very low concentration of cholesterol. As can be seen, the signal obtained from a single sensor has a very large noise, whereas the signal obtained from an average of all the sensors has a much reduced noise. Therefore, all the subsequent measurements were done using the average of all the photodiodes. Furthermore, chip-to-chip variation was also evaluated using seven different chips and found to have a standard deviation of $5.8 \mathrm{mV}$. This slight difference is due to CMOS process parameter variations, which we minimised by using control measurement as a reference for each set of measurements as demonstrated in Section D.

\section{Spectrophotometric Measurement to Different Cholesterol Concentrations}

In this section, we have quantified cholesterol in solution and serum using our proposed enzyme assay, which was measured with a bench top spectrophotometer. Initially, enzymatic activity of cholesterol oxidase was evaluated using a coupled colorimetric assay in which o-Dianisidine, in the presence of peroxidase and $\mathrm{H}_{2} \mathrm{O}_{2}$ (a product of cholesterol oxidase), is oxidised. The absorbance of the oxidised o-Dianisidine was monitored at $505 \mathrm{~nm}$ (similar to the LED peak) and $25^{\circ} \mathrm{C}$ with a UV-2550 Shimadzu Spectrophotometer. 0.5\% (w/v) cholesterol with $10 \%$ (w/v) Triton ${ }^{\mathrm{TM} X}-100$ was first made up as stock, which is equivalent to $13 \mathrm{mM}$. All experiments were performed using a cuvette with $1 \mathrm{ml}$ of solution and the enzyme assay mix is shown in Table 1. Absorbance measurements as a function of time for different cholesterol concentrations were then obtained using the spectrophotometer. The data in Fig. 5a show clearly that each absorbance levels have well differentiated magnitudes. As the substrate concentration is increased so is the final absorbance value. The velocity of the enzyme reaction was therefore measured by quantifying the amount of product formed per minute during the initial stage of the reaction, in order to extract Michaelis-Menten constant, which was $40 \pm 7 \mu \mathrm{M}$. Using a)

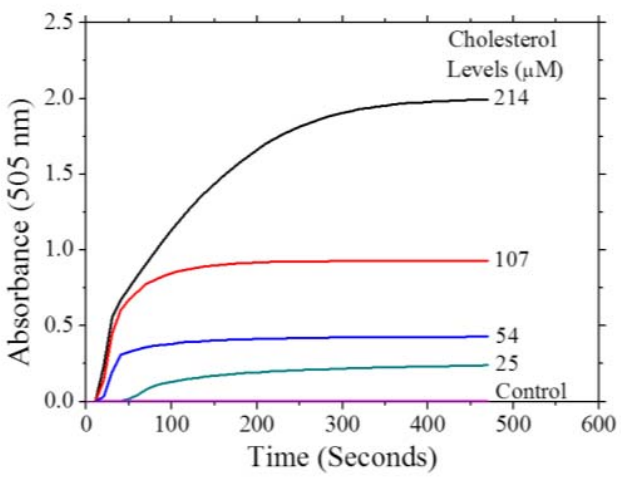

b)

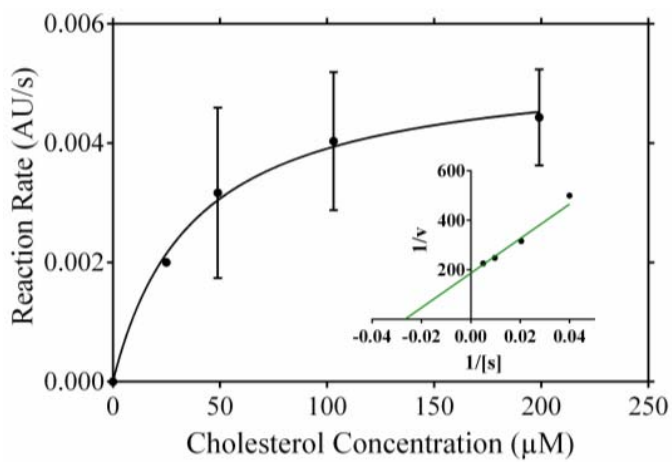

Fig. 5. a) Spectrophotometer measurement shows the absorption of oxidised o-Dianisidine for different cholesterol concentration at wavelength of $505 \mathrm{~nm}$. b) Velocity measurement of the enzyme reaction and the insert shows Lineweaver-Burk double reciprocal plot of the enzyme reaction.

TABLE I

EnZyme Assay Mix for ChOlesterol Assay

\begin{tabular}{lcccccc}
\hline \multicolumn{1}{c}{ Reagents } & Concentration & \multicolumn{5}{c}{ Volume $(\boldsymbol{\mu l})$} \\
\hline $\begin{array}{l}\text { Triethanolamine } \\
\text { (pH 7.5) }\end{array}$ & $400 \mathrm{mM}$ & 861 & 869 & 873 & 875 & 877 \\
Cholesterol & $13 \mathrm{mM}$ & 16.5 & 8.25 & 4.12 & 2.1 & 0 \\
$\begin{array}{l}\text { O-Dianisidine } \\
\text { Peroxidase }\end{array}$ & $0.32 \mathrm{mM}$ & & & 40 & & \\
$\begin{array}{l}\text { Cholesterol } \\
\text { oxidase }\end{array}$ & $3.3 \mathrm{Units}$ & & & 50 & & \\
$\begin{array}{l}\text { Total volume }(\mathrm{ml}) \\
\text { Cholesterol concentration }(\boldsymbol{\mu M})\end{array}$ & $0.33 \mathrm{Units}$ & & & 33 & & \\
\hline
\end{tabular}

the velocity and cholesterol concentration, the reaction rate versus concentration plot is presented in Fig. 5b. From the data, Lineweaver-Burk double reciprocal plot (shown in the inset of Fig. 5b) is also created using the inverse of the velocity versus inverse of the cholesterol concentration.

This was then moved on to measure the different dilutions of serum, which correspond to different cholesterol concentrations in solution. The serum was first pre-treated with cholesterol esterase for 10 minutes at $37^{\circ} \mathrm{C}$ to release free cholesterol from the esterified form prior to a reaction taking place. Serum was added to an assay mix containing $34 \mathrm{mM}$ triethanolamine $\mathrm{pH} 7.5,0.25 \%(\mathrm{w} / \mathrm{v})$ taurocholic acid sodium salt hydrate, $0.25 \%(\mathrm{w} / \mathrm{v})$ sodium cholate hydrate, $0.14 \%(\mathrm{w} / \mathrm{v})$ sodium chloride and 0.14 Units cholesterol esterase. Following preincubation, $0.32 \mathrm{mM}$ o-Dianisidine, 3 Units of peroxidase 


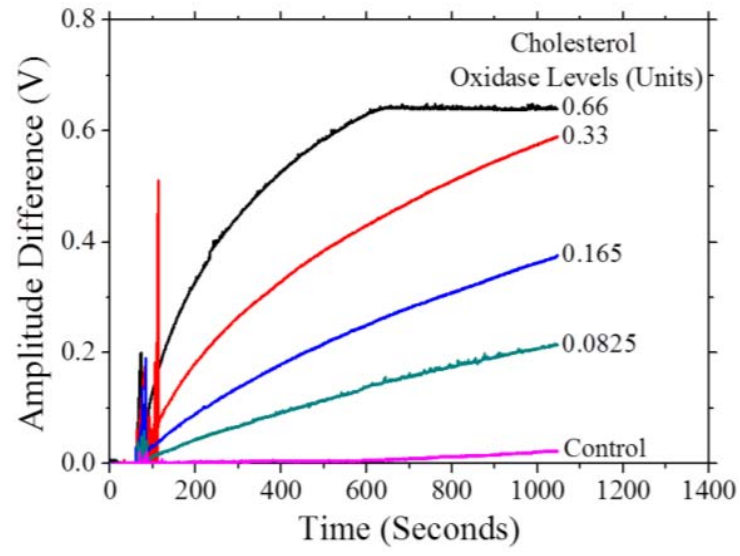

Fig. 6. Activity of cholesterol oxidase with different amounts of enzyme for a fixed amount of cholesterol. This experiment was used to estimate a good value of enzyme concentration for the subsequent cholesterol detection trials. The control measurement (no cholesterol) shows no response as expected. The initial transients are caused by the mixing of the solution when the cholesterol oxidase is added.

and 0.3 Units of cholesterol oxidase were added, and the absorbance of the oxidised o-dianisidine was monitored at $505 \mathrm{~nm}$ and $37^{\circ} \mathrm{C}$ with a UV-2550 Shimadzu Spectrophotometer. As expected, the results from different serum dilutions agree well with the results from cholesterol made in solution (data not shown).

\section{Optimizing Enzyme Concentration}

We first introduced a control experiment to define a reference baseline for our measurements. This was then followed by testing a number of cholesterol oxidase concentrations to identify which one would give an optimal measurable response within a short-time period, and which could be used for subsequent experiments. This was initially tested by spectrophotometry and later confirmed on the photodiode.

Triethanolamine buffer, o-Dianisidine, cholesterol (prepared as a solution in Triton $\left.{ }^{\mathrm{TM}} \mathrm{X}-100\right)$ and peroxidase were added to the sensor at concentrations described above. The final cholesterol concentration used in this assay was $0.429 \mathrm{mM}$ $(17.5 \mathrm{mg} / \mathrm{dl})$, as recommended by Sigma. The reaction was initiated immediately by the addition of cholesterol oxidase in equivalent reaction volumes. The optical path length from the sensor surface to the photodiode was therefore the same. The voltage change observed in Fig. 6 is a direct result of increasing absorbance over time. The sharp increase in measured voltage immediately after the peroxidase was a result of the solution being mixed, a transient effect. The photodiode chip is very robust and easily withstands repeated cleaning and reuse by rinsing with acetone, isopropanol and DI water and blow-drying with nitrogen gas. The data shown in this paper was taken from one chip for consistency.

As expected, the higher the concentration of cholesterol oxidase added the more rapid the response, with 0.66 Units of cholesterol oxidase reaching saturation level of approximately $0.63 \mathrm{~V}$, as shown in Fig. 6. At lower concentrations of cholesterol oxidase, the voltage increased more slowly, but did tend towards the same final absorbance (and voltage). The absence of cholesterol (control experiment) gave no a)

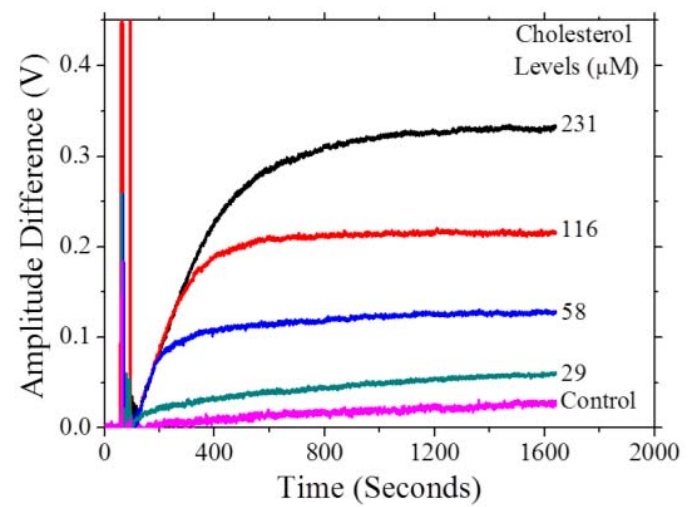

b)
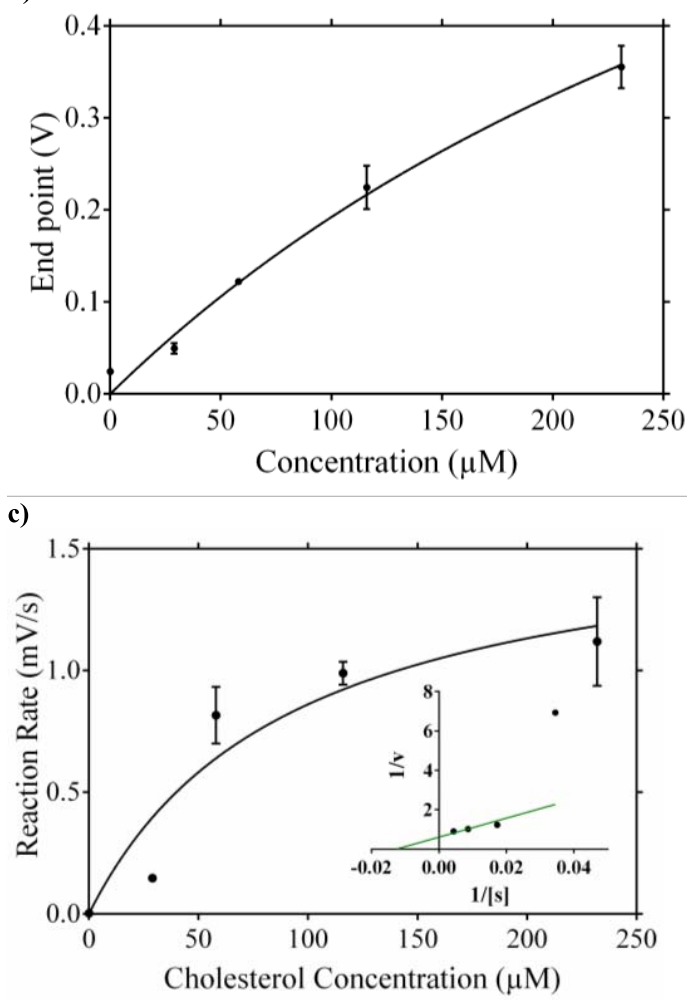

Fig. 7. a) Photodiode response data as a function of cholesterol concentration for a fixed enzyme concentration. The sensor displays good separation between different concentrations of cholesterol. The control, where no cholesterol is added, shows no response. The initial transients are caused by the mixing of the solution when the cholesterol oxidase is added. b) The endpoint voltage level as a function of cholesterol concentration. c) The velocity measurement of the enzyme reaction in serum. In the insert, we show the Lineweaver-Burk double reciprocal plot.

response, confirming that the sensor was substrate specific. This experiment validated the sensor as a means of measuring cholesterol oxidase enzyme activity and allowed us to select a concentration of 0.33 Units of cholesterol oxidase for subsequent experiments, as this concentration gave a quick temporal response and reliable measurement.

\section{E. Measurement of Photodiode Response to Different Cholesterol Concentrations in Serum}

The photodiode's response to cholesterol present in serum was then measured, by first pretreating the serum with cholesterol esterase followed by the addition of cholesterol oxidase. 
Serum was supplied at a stated cholesterol concentration of between $2.8 \mathrm{mM}-5.4 \mathrm{mM}(110 \mathrm{mg} / \mathrm{dl}-210 \mathrm{mg} / \mathrm{d})$. Using absorption method, the exact value was determined to be $3.5 \mathrm{mM}$ by the National Health Service's (NHS) laboratories of The Queen Elizabeth University Hospital, Glasgow. The stock serum was diluted in triethanolamine buffer to specific final concentrations so they were within the detectable range of the photodiode.

Our data shows that the CMOS photodiode/LED sensor system yields variable response to different cholesterol concentrations, as shown in Fig. 7a. Each of the cholesterol concentration measurements were repeated three times for consistency and repeatability using the same chip. The behaviour we observed was a good match with the spectrophotometer data (Fig. 5) indicating that the assay works well on the CMOS chip and that the miniature optical measurement system is comparable to conventional lab-based spectrophotometry. The voltage readout increased monotonically with cholesterol concentration. No change in voltage was observed when cholesterol was omitted. Each reaction reached a different final plateau voltage, signifying consumption of substrate and corresponding to the substrate concentration used.

Endpoint voltages as a function of final cholesterol concentration is plotted in Fig. 7b. As can be noticed, the lowest cholesterol concentration in pure human blood serum that we measured is at $29 \mu \mathrm{M}$. The limit of detection of the assay is also determined by taking three times of the standard deviation from control experiment, which is approximately $13 \mu \mathrm{M}$. The total cholesterol in human blood for a healthy individual should be $5 \mathrm{mM}$ or lower [28], hence our device has the potential to be also used for more sensitive assays.

Similar to data analysis used for the spectrophotometer, the initial velocity was plotted against cholesterol concentrations in serum to calculate the $K_{M}$ value. $K_{M}$ was found to be $80 \pm 1 \mu \mathrm{M}$ using the new method. The data was obtained using the velocity versus concentration plot and LineweaverBurk double reciprocal plot in Fig. 7c. The result is in good agreement with the value obtained from the spectrophotometer. The work has therefore demonstrated that a low-cost and simple green LED/photodiode system has the capability to replicate the quantification of cholesterol in serum as a bench top spectrophotometer.

\section{CONCLUSION}

Elevated cholesterol is associated with an increased risk of developing cardiovascular disease with resultant high levels of morbidity and mortality. Monitoring the cholesterol levels of a patient in a point-of-care setting would make significant progress towards controlling cardiovascular disease, and would help save the lives of those affected, with a concomitant reduction in healthcare costs. Currently spectrophotometry is the gold standard used to determine cholesterol levels. Here, a method to determine the relative amounts of cholesterol in serum has been demonstrated using a miniaturised derivation from the spectrophotometric assay, by using a CMOS system. The assay method has been shown to be sensitive enough to detect serum cholesterol levels in a concentration dependent manner spanning the range of $29 \mu \mathrm{M}$ to $231 \mu \mathrm{M}$, and therefore has the potential to be further developed as a practical handheld sensor device. Future challenges will include creating an assay sample technology that will allow the fractionation of blood so that serum can be added onto the sensor.

The use of a CMOS sensor allows the device to be interfaced to electronic devices and displays, which could lead to more functional and practical devices using mobile computing. Such devices could be more easy to use and reliable than teststrips that often lack a good user interface. The results show excellent potential for the development of an early-warning device for the detection of an individual's elevated cholesterol - leading to a low-cost and commercial diagnostic tool for home cholesterol management, which could subsequently spur the development of similar products for other metabolomic health indicators using different enzyme assays. In the long term we anticipate such devices will play a major role in metabolomic science and precision medicine whereby personalized healthcare is made possible through the use of precise patient data.

\section{NOTES}

*Low density lipoprotein cholesterol, often termed "bad cholesterol".

\section{REFERENCES}

[1] P. Yager, G. J. Domingo, and J. Gerdes, "Point-of-care diagnostics for global health," Annu. Rev. Biomed. Eng., vol. 10, pp. 107-144, Jan. 2008.

[2] I. V. Jani and T. F. Peter, "How point-of-care testing could drive innovation in global health," New England J. Med., vol. 368, no. 24 pp. 2319-2324, Jun. 2013.

[3] D. A. Giljohann and C. A. Mirkin, "Drivers of biodiagnostic development," Nature, vol. 462, pp. 461-464, Nov. 2009.

[4] A. P. F. Turner, "Biosensors: Sense and sensibility," Chem. Soc. Rev, vol. 42, no. 8, pp. 3184-3196, Apr. 2013.

[5] C. D. Chin et al., "Microfluidics-based diagnostics of infectious diseases in the developing world," Nature Med., vol. 17, no. 8, pp. 1015-1019, Aug. 2011.

[6] X. Zhang, H. Ju, and J. Wang, Electrochemical Sensors, Biosensors and Their Biomedical Applications. San Diego, CA, USA: Academic, 2008, pp. 57-67.

[7] M. S. Monteiro, M. Carvalho, M. L. Bastos, and P. G. De Pinho, "Metabolomics analysis for biomarker discovery: Advances and challenges," Current Med. Chem., vol. 20, no. 2, pp. 257-271, Jan. 2013.

[8] A. Nordström and R. Lewensohn, "Metabolomics: Moving to the clinic," J. Neuroimmune Pharmacol., vol. 5, no. 1, pp. 4-17, Mar. 2010.

[9] E. S. Ford, W. H. Giles, and W. H. Dietz, "Prevalence of the metabolic syndrome among US adults findings from the third national health and nutrition examination survey," J. Amer. Med. Assoc., vol. 287, no. 3, pp. 356-359, 2002.

[10] Scandinavian Simvastatin Survival Study Group, "Randomised trial of cholesterol lowering in 4444 patients with coronary heart disease: The scandinavian simvastatin survival study (4S)," Lancet, vol. 344 , no. 8934, pp. 1383-1389, Nov. 1994.

[11] J. R. Hupp, "Lowering blood cholesterol to prevent heart disease," J. Oral Maxillofac. Surgery, vol. 43, no. 9, p. 741, Sep. 1985.

[12] M. L. Stefanick, S. Mackey, M. Sheehan, N. Ellsworth, W. L. Haskell, and P. D. Wood, "Effects of diet and exercise in men and postmenopausal women with low levels of HDL cholesterol and high levels of LDL cholesterol," New England J. Med., vol. 339, no. 1, pp. 12-20, 1998.

[13] M. J. Martin, W. S. Browner, S. B. Hulley, L. H. Kuller, and D. Wentworth, "Serum cholesterol, blood pressure, and mortality: Implications from a cohort of 361662 men," Lancet, vol. 328, no. 8513, pp. 933-936, 1986.

[14] D. Mozaffarian et al., "Heart disease and stroke statistics-2015 update," Circulation, vol. 131, no. 4, pp. e29-e322 , Jan. 2015.

[15] A Global Brief on Hypertension-Silent Killer, Global Public Health Crisis, Switzerland: World Health Organization, 2013.

[16] E.-H. Yoo and S.-Y. Lee, "Glucose biosensors: An overview of use in clinical practice.," Sensors, vol. 10, no. 5, pp. 4558-4576, Jan. 2010. 
[17] L. Sommer, Analytical Absorption Spectrophotometry in the Visible and Ultraviolet. New York, NY, USA: Elsevier, 1989.

[18] J. Monk, "Developments in microelectronics technology and the economics of the semiconductor industry," Int. J. Soc. Econ., vol. 7, no. 1, pp. 13-23, 1980.

[19] D. Lombardo and O. Guy, "A rapid and convenient colorimetric assay for cholesterol ester hydrolase activity," Biochimie, vol. 61, no. 3, pp. 415-417, Jun. 1979.

[20] Y. Kayamori, H. Hatsuyama, T. Tsujioka, M. Nasu, and Y. Katayama, "Endpoint colorimetric method for assaying total cholesterol in serum with cholesterol dehydrogenase," Clin. Chem., vol. 45, no. 12, pp. 2158-2163, Dec. 1999.

[21] S. Singh, P. R. Solanki, M. K. Pandey, and B. D. Malhotra, "Cholesterol biosensor based on cholesterol esterase, cholesterol oxidase and peroxidase immobilized onto conducting polyaniline films," Sens. Actuators B Chem., vol. 115, no. 1 , pp. 534-541, May 2006.

[22] W. Richmond, "Preparation and properties of a cholesterol oxidase from Nocardia sp. and its application to enzymatic assay of total cholesterol in serum," Clin. Chem., vol. 19, no. 12, pp. 1350-1356, 1973.

[23] P. Srisawasdi, N. Prasertsincharoen, and M. H. Kroll, "Porcine pancreas: A superior source of cholesterol esterase for total serum cholesterol assay by the enzymatic kinetic method," J. Clin. Lab. Anal., vol. 26, no. 6, pp. 420-430, Nov. 2012

[24] C. C. Allain, L. S. Poon, C. S. Chan, W. Richmond, and P. C. Fu, "Enzymatic determination of total serum cholesterol," Clin. Chem., vol. 20, no. 4, pp. 470-574, Apr. 1974.

[25] J. R. Harris, Cholesterol Binding and Cholesterol Transport Proteins. New York, NY, USA: Springer, 2010.

[26] E. R. Fossum, "CMOS image sensors: Electronic camera-on-a-chip," IEEE Trans. Electron Devices, vol. 44, no. 10, pp. 1689-1698, Oct. 1997.

[27] B. C. Cheah et al., "An integrated circuit for chip-based analysis of enzyme kinetics and metabolite quantification," IEEE Trans. Biomed. Circuits Syst., vol. 10, no. 3, pp. 721-730, Jun. 2016.

[28] S. D. Goodman et al., "Report of the national cholesterol education program expert panel on detection, evaluation, and treatment of high blood cholesterol in adults," Arch. Int. Med., vol. 148, no. 1, pp. 36-69, 1988.

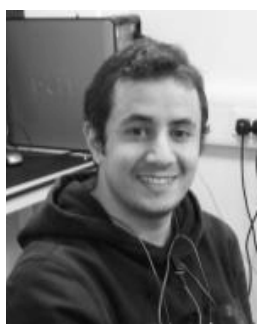

Mohammed A. Al-Rawhani received the B.Sc. degree in electronics and telecommunication engineering from Amman University, Amman, Jordan, in 2004, and the M.Sc. degree in electrical and electronic engineering and the Ph.D. degree in electronic integration design for biomedical applications from the University of Glasgow, Glasgow, U.K., in 2007 and 2012, respectively. His research interests include CMOS low- and highvoltage analogue/mixed-signal integrated circuits and front-ends for optical sensing biomedical

applications.

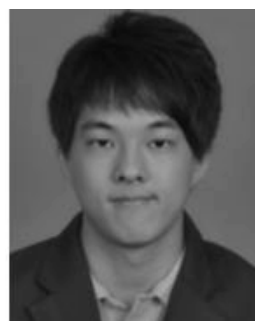

Boon Chong Cheah received the B.Eng. degree in electronic and electrical engineering and the M.Sc (Eng.) degree in nanotechnology and advanced electronic devices from the University of Leeds, Leeds, U.K., in 2010 and 2011 respectively. He is currently pursuing the Ph.D. degree in electronic and electrical engineering with the University of Glasgow, Glasgow, U.K., where he is with the Microsystem Technology Group.

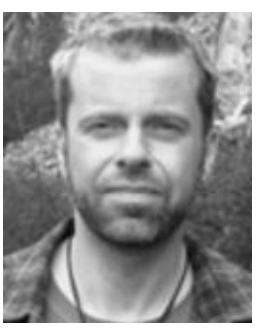

Alasdair Iain Macdonald received the B.Sc (Hons.) degree in molecular biology from the University of Glasgow, Glasgow, U.K., and the Ph.D. degree from the Wellcome Trust. He is currently with the Institute for Infection, Immunity, and Inflammation, University of Glasgow, where he is developing enzyme assays on biosensor devices.

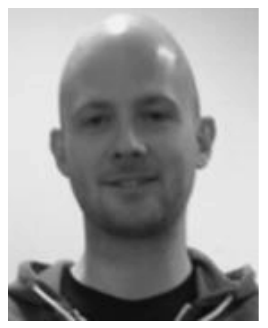

Christopher Martin received the M.Eng. degree in electronics and electrical engineering and the Ph.D. degree in electronics and nanoscale engineering from the University of Glasgow, Glasgow, U.K., in 2009 and 2013, respectively. He is currently a PostDoctoral Research Assistant. His interests center on peripheral nerve repair strategies, wireless and flexible electronics, printing techniques, electrochemistry, and bioelectronics.

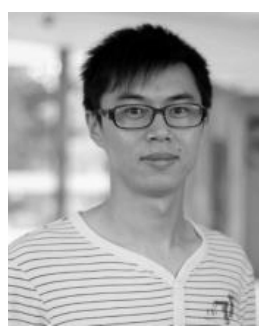

Chunxiao Hu received the B.Sc. degree in automation in China in 2008, the M.Sc. degree in microsystem technology in Southampton in 2009, and the $\mathrm{Ph}$.D. degree in microfluidic devices for nematodes in Southampton in 2013. His research interests include lab on chip, microfluidics, C. elegans, platform integration, nanowire/nanoribbon, surface functionalization, and biosensing. His research currently mainly focuses on using CMOS-based chemical sensors for multiple detection.

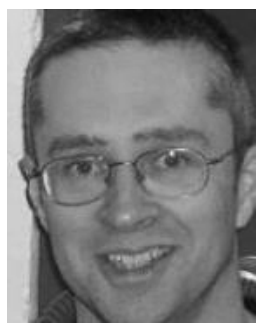

James Beeley received the B.Eng. and Ph.D. degrees from the University of Glasgow, Glasgow, U.K., in 1997 and 2003, respectively. He is currently with the University of Glasgow. His research interests include parallel computer hardware design, very large scale integration design, and electronic.

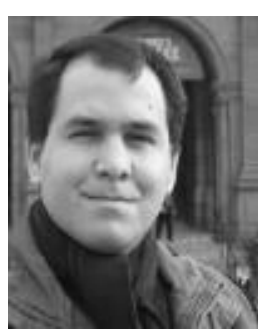

Luiz Carlos Gouveia received the B.Sc. and M.Sc. degrees from the Federal University of Rio de Janeiro, Rio de Janeiro, Brazil, in 1995 and 2001, respectively, and the Ph.D. degree from the University of Edinburgh, U.K., in 2011, all in electrical engineering. His research interests span from low-power analogue circuits to programmable ana$\log$ VLSI to image sensor systems to bioinspired electronic systems.

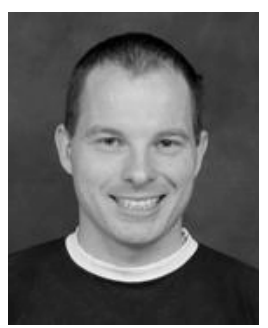

James P. Grant received the B.Sc. and Ph.D. degrees in physics from the University of Glasgow, Glasgow, U.K., in 2002 and 2006, respectively. He is currently a Post-Doctoral Research Fellow with the School of Engineering, University of Glasgow, where his research interests include nanofabrication, metamaterial devices, plasmonics, terahertz systems, sensors, imaging, and CMOS electronics.

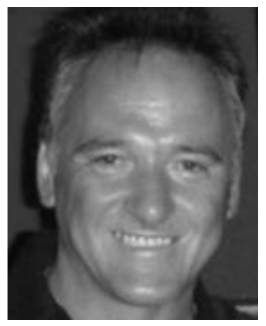

Gordon Campbell has provided technical research support at the University of Glasgow, Glasgow, U.K., since 1972. He has research expertise in cell biology, cell engineering, microbiology, and parasitology. 


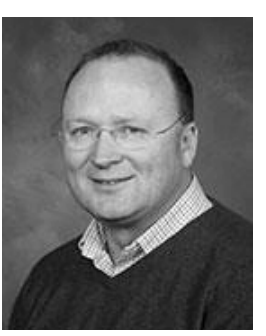

Michael P. Barrett is currently a Professor of Biochemical Parasitology with the University of Glasgow, Glasgow, U.K., and directs the Glasgow Polyomics and the Scottish Metabolomics Facilities. $\mathrm{He}$ is also the Deputy Director of the Scottish Universities Life Sciences Alliance and also an Associate Director of the U.S.-based Consortium of Parasitic Drug Development. He chaired the Kinetoplastids drug efficacy working group at the World Health Organization. He has pioneered application of novel technologies to diagnostics and new antimicrobial interventions and the use of microfluidics for separation of parasites from mammalian body fluids.

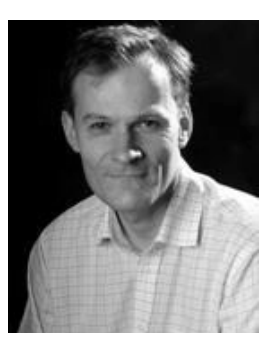

David R. S. Cumming (M'97-SM'08-F'13)

received the B.Eng. degree in electronic and electrical engineering from the University of Glasgow, Glasgow, U.K., in 1989, and the Ph.D. degree from the Cambridge University, Cambridge, U.K., in 1993. He currently leads the Microsystem Technology Group, School of Engineering, University of Glasgow, where he is also the Co-Founder of its spin-out, Mode Diagnostics. His research on CMOS ion sensitive array technology has also been commercialized into the ion torrent next-generation sequencing system. He was a recipient of the Royal Society Wolfson Merit Award. 